\title{
Dispersion and energy compensation in high-gradient linacs for lepton colliders
}

\author{
Roger M. Jones, ${ }^{1,2, *}$ Valery A. Dolgashev, ${ }^{3}$ and Juwen W. Wang ${ }^{3}$ \\ ${ }^{1}$ School of Physics and Astronomy, The University of Manchester, Oxford Road, Manchester, M13 9PL, United Kingdom \\ ${ }^{2}$ Cockcroft Institute of Science and Technology, Daresbury, WA4 4AD, United Kingdom \\ ${ }^{3}$ SLAC National Accelerator Laboratory, Menlo Park, California 94025, USA
}

(Received 26 February 2009; published 29 May 2009)

\begin{abstract}
The shape of an rf pulse is distorted due to dispersion encountered in acceleration through travelingwave linear accelerator structures. Simulations are made to ascertain the severity of this distortion in cavities designed to operate at various group velocities. The pulse suffers maximum degradation when propagated through accelerating cavities with a phase advance per cell in the vicinity of $\pi$, where the group velocity reaches its minimum value. Several cavities are simulated to study the pulse distortion and compared with experiments performed on a high phase advance structure H60VG3, which has a phase advance of $5 \pi / 6$ per cell. A circuit model and a mode matching code are used to perform these simulations on accelerating structures consisting of multiple cells. Beam loading is taken into account and the implications on energy dispersion are also analyzed. Means of mitigating for this energy deviation are discussed.
\end{abstract}

DOI: 10.1103/PhysRevSTAB.12.051001

PACS numbers: 29.20.Ej, 41.75.Fr, 41.75.Ht

\section{INTRODUCTION}

In accelerating a charged particle beam to ultrarelativistic energies, linear rather than circular accelerators are needed. In the context of the next generation of linear colliders [1-6], several thousand of these cavities will be required. At the SLAC National Accelerator Laboratory, cavities operating with a $2 \pi / 3$ phase advance per cell at an $S$-band frequency of $2.856 \mathrm{GHz}$ are able to reach a stable gradient of approximately $20 \mathrm{MV} / \mathrm{m}$ enabling the beam to be accelerated to an energy of approximately $60 \mathrm{GeV}$ [7] along a linac of length $3 \mathrm{~km}$. There are approximately 960 structures in the linac, each of which is $3.05 \mathrm{~m}$ long (10 ft) and houses 86 cells (including cell 1 and 86, the coupler cells) [8]. The monopole mode used to accelerate the beam has a finite group velocity which decreases along the structure in order to preserve a constant gradient [8]. The variation of group velocity with frequency corresponds to the dispersion which the accelerating pulse applied to the beam will encounter $[9,10]$. For the SLAC two-mile linac the dispersion was manageable.

However, in order to achieve higher energies and higher luminosities at collision, the next generation of normal conducting (NC) linear colliders, such as that adopted by the CLIC $[5,6]$ project, is aiming at an ambitious loaded gradient of $100 \mathrm{MV} / \mathrm{m}$ at an $X$-band accelerating frequency of $11.9942 \mathrm{GHz}$. The baseline design for the CLIC project will utilize 142812 accelerating structures [11] and each positron and electron linac will be $\sim 21 \mathrm{~km}$

\footnotetext{
*Corresponding author. Current address: School of Physics and Astronomy, The University of Manchester, Oxford Road, Manchester, M13 9PL, United Kingdom.

roger.jones@manchester.ac.uk
}

long. The center of mass energy at collision is expected to be $3 \mathrm{TeV}$ for the current design and with a peak luminosity of $7 \times 10^{34} \mathrm{~cm}^{-2}$ [6]. In order to mitigate for the effects of breakdown issues in these structures, cavities with different group velocities are being intensively investigated by focused groups at CERN, KEK, and the SLAC National Laboratory in particular. Several structure designs are under intensive investigation and those with a phase advance per cell of $2 \pi / 3$ and $5 \pi / 6$ have been investigated both experimentally and theoretically to explore the physics of fundamental breakdown limitations and to ascertain their suitability of being used routinely within the CLIC collider. These structures have capitalized on the valuable experience gained with the wealth of structures built at SLAC, KEK, and FNAL during the Next Linear Collider/ Global Linear Collider (NLC/GLC) research program [4] which aimed at a loaded gradient of $55 \mathrm{MV} / \mathrm{m}$ at an accelerating frequency of $11.424 \mathrm{GHz}$. One recent CLIC prototype structure consisting of 18 cells is illustrated in Fig. 1, and is referred to as T18_VG2.6_disk [12]. The irises in each of the cells are reduced in order to limit the surface field on the walls of the irises and the group velocity tapers down from $0.028 c$ to $0.01 c$ at a phase advance per cell of $2 \pi / 3$. Additional structures are being investigated with cell phase advances of $5 \pi / 6$. The original motivation for investigating high phase advance structures was based on observation of breakdown which was occurring in the NLC/GLC structure known as RDDS1, in which significant damage to the cells was observed on the high group velocity $(0.12 c)$ end of the structure but minimal damage on the low group velocity $(0.03 c)$ end. Choosing a phase advance per cell closer to $\pi$ is one means of reducing the group velocity. The CLIC project maintains high phase advance structures as an option for the baseline 


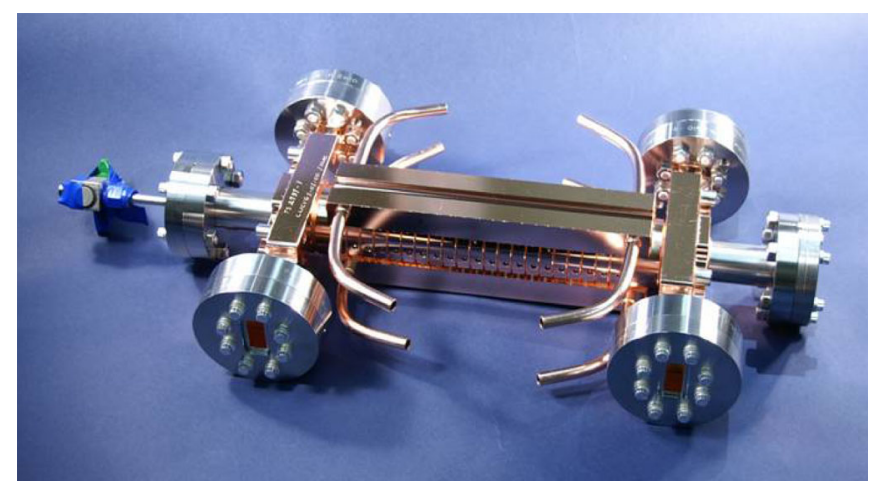

FIG. 1. (Color) Accelerating structure T18_VG2.6_disk [12] with fundamental power couplers attached.

design as a final decision for specific structure has not been decided upon. An example of such a high phase advance structure, consisting of 55 cells and $60 \mathrm{~cm}$ in length, is illustrated in Fig. 2. The group velocity in this structure varies from $0.05 c$ to $0.008 c$. The distortion in an rf pulse propagated through these structures is expected to be more severe as the phase advance per cell is increased as the group velocity is reduced and as the relative frequency bandwidth is reduced. The ratio of the maximum surface electric field to that of the accelerating field has been maintained at approximately 2 throughout this structure.

In this paper the dispersion characteristics of these linac structures are investigated. The group velocity in linacs depends on frequency and thus each frequency component will travel at a different speed through the structure. This will inevitably give rise to distortion in the shape of an $\mathrm{rf}$ pulse propagated through the structure. An rf electromagnetic pulse is used to accelerate a bunch train of charged particles. The motivation of this work is to understand the distortion expected to occur in the shape of the rf pulse and the corresponding energy dispersion which will occur along the accelerated bunch train. Shaping the rf pulse which is delivered to the accelerator by suitably phasing the klystrons is investigated as a means of compensating for this energy dispersion.

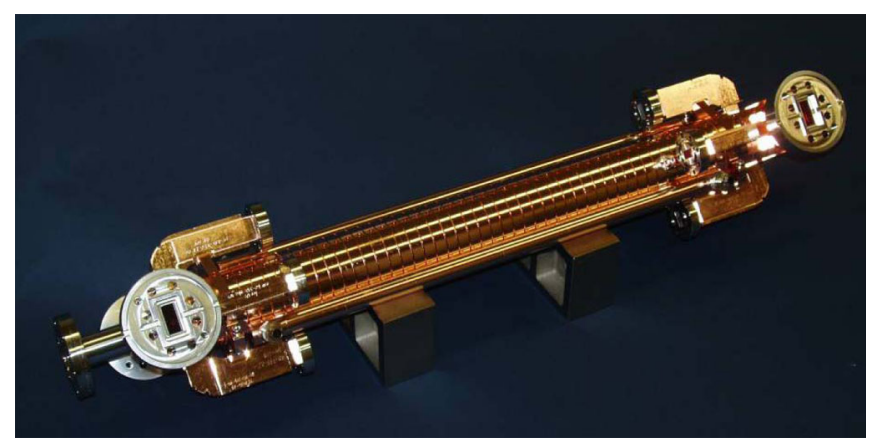

FIG. 2. (Color) High phase advance accelerating structure, H60VG4SL17A/B [28].
This paper is organized such that the steady state beam loading expected in NLC/GLC structures is described in the next section from energy balance considerations. The second section entails both a circuit description and a mode matching code analysis. The third section provides detailed simulations of pulse propagation through a series of accelerating structures and is compared with experimental results. The fourth section entails an analysis of the distortion in the energy over the train of accelerated bunches. The final section provides some conclusions on the dispersive effects in these traveling-wave structures.

\section{STEADY STATE BEAM LOADING}

The rf accelerating field loses energy to both the charged particle beam and to the walls of the accelerating cavity, and for a normal conducting cavity the latter is a significant fraction of the total energy loss. The power flow per unit length in terms of the cavity $Q$ is given as

$$
\frac{d P}{d z}=-\frac{\omega P}{v_{g} Q}
$$

where the energy per unit length $U^{\prime}$ flows with group velocity $v_{g}$ and $P=U^{\prime} v_{g}$. In a linear collider, in order to optimize efficiency, a train of bunches is accelerated. The energy lost by a charged particle bunch is characterized by a loss factor [13] which denotes the energy lost per unit charge $q$ squared. The corresponding loss factor per unit length is given by

$$
k^{\prime}=\left(\frac{\omega R^{\prime}}{4 Q}\right)=-\frac{1}{2} \frac{v_{g}}{I_{c}} \frac{d E}{d z},
$$

where $I_{c}$ is the beam current, $E$ the electric field in the cavity, and $R^{\prime}$ denotes the shunt impedance per unit length [13]. The latter provides a measure of the efficiency of coupling the rf power to the beam:

$$
R^{\prime}=\frac{E^{2}}{-\frac{d P}{d z}}
$$

This enables the power to be obtained as

$$
P=\frac{v_{g} E^{2}}{\left(\frac{\omega R^{\prime}}{Q}\right)} .
$$

Differentiating with respect to $z$ gives the incremental power flow:

$$
d P=\frac{2 v_{g} E d E}{\left(\frac{\omega R^{\prime}}{Q}\right)}=-\sqrt{\frac{\omega R^{\prime}}{v_{g} Q}} I_{c} P^{1 / 2} d z,
$$

where Eqs. (2) and (4) have been used. Thus, gathering both loss terms presented in Eqs. (1) and (5) we obtain

$$
\frac{d P}{d z}+\frac{\omega}{v_{g} Q} P+\sqrt{\frac{\omega R^{\prime}}{v_{g} Q}} I_{c} \sqrt{P}=0 .
$$


This first order differential equation may be solved to obtain the power flow once $R, v_{g}, Q$, and $I_{c}$ have been specified. In the structures being designed for the next generation of linear colliders all of these quantities, apart from the beam current, are a function of distance $z$ along the accelerating axis. However, for constant coefficients Eq. (6) is readily solved:

$$
\begin{aligned}
P_{\text {app }}(z)= & {\left[I_{c} Q \sqrt{\frac{R^{\prime} / Q v_{g}}{\omega}}\left(1-e^{-\left[\omega /\left(2 Q v_{g}\right)\right] z}\right)\right.} \\
& \left.+\sqrt{P_{0}} e^{-\left[\omega /\left(2 Q v_{g}\right)\right] z}\right]^{2} .
\end{aligned}
$$

For the sake of completeness we note that the differential equation describing the electric field is readily obtained from a substitution of Eq. (4) into Eq. (5):

$$
\frac{d}{d z}\left(\frac{E^{2} v_{g}}{R^{\prime} / Q}\right)+\frac{E^{2} \omega}{\left(R^{\prime} / Q\right) Q}+E I_{c} \omega=0
$$

For the case of constant coefficients this is also readily solved:

$$
E(z)=I_{C} R^{\prime}\left(1-e^{-\left[\omega /\left(2 Q v_{g}\right)\right] z}\right)+E_{0} e^{-\left[\omega /\left(2 Q v_{g}\right)\right] z} .
$$

However, for the structures considered for a linear collider, Eq. (8) must be solved numerically. The beam-loaded field for parameters corresponding to the accelerating structure H60VGVG3 [13] are illustrated in Fig. 3 together with that of the approximate solution given by Eq. (9) with $R^{\prime}, V_{g}, Q$ evaluated within the center of the structure. The total beam-loaded field integrated over the length of the structure gives the total beam-loading potential which in this case is $33.9 \mathrm{MV}$ or $56.3 \mathrm{MV} / \mathrm{m}$ in terms of the average beam-loaded field. Taking the approximate value from Eq. (9) and integrating over the length of the structure

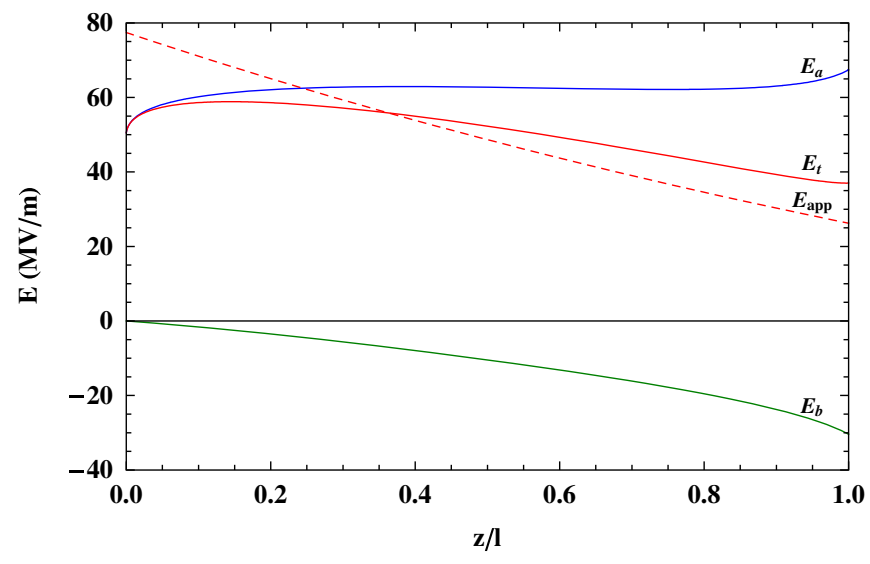

FIG. 3. (Color) Components of accelerating field in H60VG4SL17A/B. $E_{a}$ denotes the accelerating field without beam loading, $E_{t}$ the total beam-loaded field, and $E_{b}$ the difference between the total field and the accelerating field. Also shown is $E_{\text {app }}$, an approximation to the total field experienced by the beam. gives comparable values: $33.3 \mathrm{MV}$ and $55.4 \mathrm{MV} / \mathrm{m}$, respectively. Thus, the approximate form of the field with all parameters evaluated within the center of the structure provides a very good approximation to the total beamloaded potential experienced by the beam.

\section{PULSE DISTORTION DUE TO DISPERSION}

In order to model the transient effects due to dispersion in linear accelerator structures, we consider the propagation of an rf pulse through a multicell structure using two methods: a circuit model and a mode matching code, SMART2D [14]. Both approaches entail obtaining the fields in individual cells in the frequency domain and this constitutes the transfer function of the structure. Taking the Fourier transform of the transfer function and convolving with the input pulse allows the progress of a pulse to be monitored as it passes through each cell. In this section no account is taken for the beam loading, as the focus is on distortion in pulse shape due to dispersive effects resulting from the dependence of group velocity on frequency. The forthcoming Sec. IV addresses the issue of beam loading.

The mode matching method is based on a modal expansion of the electromagnetic field and entails matching the fields at transverse cross sections between transitions in waveguide [15]. Both methods give similar results on the shape of the pulse after propagating through the linacs. Matching into the periodic structures is affected by modifying the end cells. The geometry of the interior (non-end) cells, which compose the entire linac, are not modified in this process, but the degree to which the design phase advance per cell has been achieved is recorded in both methods.

First, we describe the circuit model employed to model an $\mathrm{N}$-cell chain and this is given by a series of resonant circuits inductively coupled as illustrated in Fig. 4. Three typical cells are indicated together with the end cells. The capacitance, inductance, and resistance vary along the chain corresponding to modifying the cell geometry. The mutual inductance, coupling for example cell $n-1$ and cell $n$, is given by $\kappa_{n-1 / 2}\left(L_{n-1 / 2} L_{n}\right)^{1 / 2}$. This model is similar to that developed in [16]. The current in the $n$th cell $I_{n}$ corresponds to the accelerating field evaluated in the

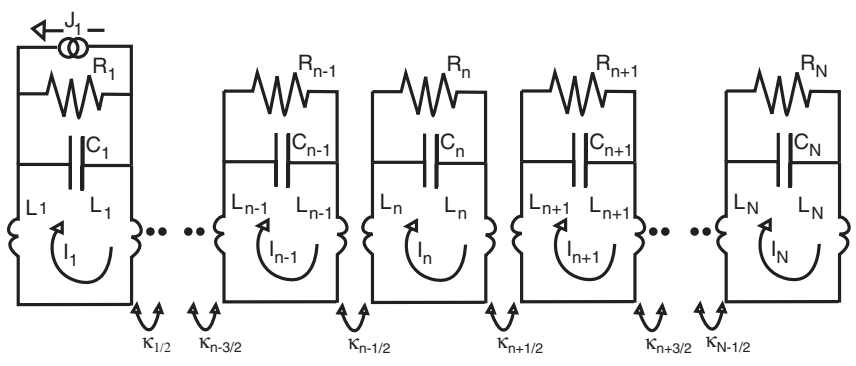

FIG. 4. Circuit model of the fundamental mode of a travelingwave linear accelerator cavity. 
center of the accelerating cell. Excluding the resistors and cell coupling, the resonant frequency of the $n$th inductorcapacitor chain is given by $\omega_{n}^{\prime} / 2 \pi=\left(2 L_{n} C_{n}\right)^{1 / 2}$. Adding the resistance corresponding to wall losses, and adding nearest neighbor coupling, will modify these cell frequen- cies and give rise to coupled mode frequencies. In order to obtain these mode frequencies, we form the Kirchhoff current loops in the frequency domain for the coupled chain and after some algebraic manipulation we obtain the following matrix equation:

$$
\left(\begin{array}{cccc}
1-\frac{\omega_{1}^{2}}{\omega^{2}} & \kappa_{1 / 2} \frac{2 \omega_{1}^{\prime} K_{2}}{2 \omega_{2}^{\prime} K_{1}} & \ldots & \cdots \\
\cdots & \cdots & \cdots \\
\cdots \kappa_{n-1 / 2} \frac{\omega_{n}^{\prime} K_{n-1}}{2 \omega_{n-1}^{\prime} K_{n}} & 1-\frac{\omega_{n}^{2}}{\omega^{2}} & \kappa_{n+1 / 2} \frac{\omega_{n}^{\prime} K_{n+1}}{2 \omega_{n+1}^{\prime} K_{n}} & \cdots \\
\cdots & \cdots & \kappa_{n-1 / 2} \frac{2 \omega_{n}^{\prime} \omega_{N-1}}{2 \omega_{N-1}^{\prime} K_{N}} & 1-\frac{\omega_{N}^{2}}{\omega^{2}}
\end{array}\right)\left(\begin{array}{c}
I_{1} \\
I_{2} \\
\cdots \\
I_{n} \\
\cdots \\
I_{N}
\end{array}\right)=\left(\begin{array}{c}
J_{1} \frac{\omega_{1}^{2}}{\omega^{2}} \\
\cdots \\
\cdots \\
\cdots \\
\cdots
\end{array}\right),
$$

where for the $n$th cell $\omega_{n}=\omega_{n}^{\prime}\left(1-i \frac{\omega_{n}^{\prime}}{Q_{n} \omega}\right)^{-1 / 2}, Q_{n}=$ $\omega_{n} C_{n} R_{n}$, and the characteristic loss factor [13] is given by $K_{n}=\frac{\omega_{n} R_{n}}{4 Q_{n}}$. The matrix multiplying the current vector is a square matrix of dimension $N \times N$. Setting the current drive $J_{1}=0$ and solving the resulting matrix equation gives $N$ eigenfrequencies $\omega / 2 \pi$ of the accelerator and this approach was followed in [16]. For example, for a structure consisting of an infinite number of identical cells, $\kappa_{n-1 / 2}=\kappa_{n+1 / 2}=\kappa$ and the Floquet condition for the $n$th cell of an infinitely periodic structure is $I_{n}=I_{0} e^{- \text {in } \phi}$. The eigenfrequencies for this infinitely periodic structure are readily determined as $\omega=\omega_{\pi / 2}(1+\kappa \cos \phi)^{-1 / 2}$, where $\phi$ is the phase advance per cell. Thus, the circuit model is parametrized by $\omega_{\pi / 2}$ the frequency at a phase advance of $\pi / 2$ and the bandwidth $\kappa$ Also, we note that in this case the matrix in Eq. (10) is independent of the loss factor $K$, as is expected when beam-loading issues are excluded from the model. The loss factor is in fact used in the model to account for adiabatic changes in cell geometry.

However, in general the cell parameters are not identical but for most situations of interest to the NLC and CLIC they vary in an adiabatic manner. The method proceeds by evaluating a set of fiducial cells with the parameters of these cells determined from the infinite periodic structure conditions. Intermediate cells are then determined by spline interpolation. In this manner multicell cavities are rapidly and accurately characterized. The driven response of the circuit to a current $J_{1}$ applied to the first cell is then obtained by solving the matrix given in Eq. (10).

For the NLC we focused on 55-cell cavities and we use this number of cells in all simulations described in this work. In order to track the progress of an rf accelerating pulse through the multicell cavity, we take the convolution of the time response of the circuit with a specified rf pulse shape and this corresponds to the inverse Fourier transform of the product of the frequency response of the circuit with the Fourier transform of the applied rf signal. Prior to performing this analysis of pulse propagation, we ensure the input and output cells of the circuit are properly matched such that an acceptable transmission occurs within the band of the accelerating frequency and the field within the cavity is flat to maximize the efficiency of acceleration.

There are two main approaches we have adopted to achieve this tuning of the cavity. The first method, which we will refer to as the direct method, entails an evaluation of the reflection coefficient $S_{11}$ at the input drive port and varies the input cell frequency and coupling factor such that $\left|S_{11}\right|$ is minimized at the accelerating frequency and several frequencies nearby. The second method is based on the analysis of Kroll et al. [16] and requires three current (representative of the electric field in [17]) points spaced by $P$ one cell period. In this work we focus our effort on the latter method. The current within the circuit is constructed from a forward traveling wave $I(z) e^{-i \psi(z)}$ and a reflected wave $I(z) R e^{i \phi(z)}$ and, hence, the total current at any location $z$ within the cavity is given by

$$
I_{c}=I(z)\left[e^{-i \psi(z)}+R e^{i \psi(z)}\right]
$$

From the analysis of [16] we obtain

$$
\begin{aligned}
\cos \phi & =\frac{I_{c}(z+P)+I_{c}(z-P)}{2 I_{c}(z)}, \\
\operatorname{Re}^{2 i \psi} & =\frac{2 \sin \phi-i I_{c}(z+P)+i I_{c}(z-P)}{2 \sin \phi+i I_{c}(z+P)-i I_{c}(z-P)},
\end{aligned}
$$

where $\psi$ is a real valued phase, periodic, except for a cell to cell phase advance $\phi$ :

$$
\psi(z \pm P)=\psi(z) \pm \phi
$$

and $R$ is a reflection coefficient. Both $R$ and $\psi$ vary along the structure. We apply this method to several structures and minimize the reflection coefficient within the structure in each case. Once the Kroll method has been applied, we verify its accuracy by applying the direct method.

This technique is applied to a 55 cell structure composed of identical cells each with a phase per cell of $2 \pi / 3$ and a monopole mode group velocity of $0.03 c$. We refer to this $60 \mathrm{~cm}$ long structure as CZ120VG3. After iteratively tuning the input and output cells by the Kroll method, the resulting mean interior reflection coefficient is $-95 \mathrm{~dB}$ and mean phase advance per cell is $120.01^{\circ}$. Applying 


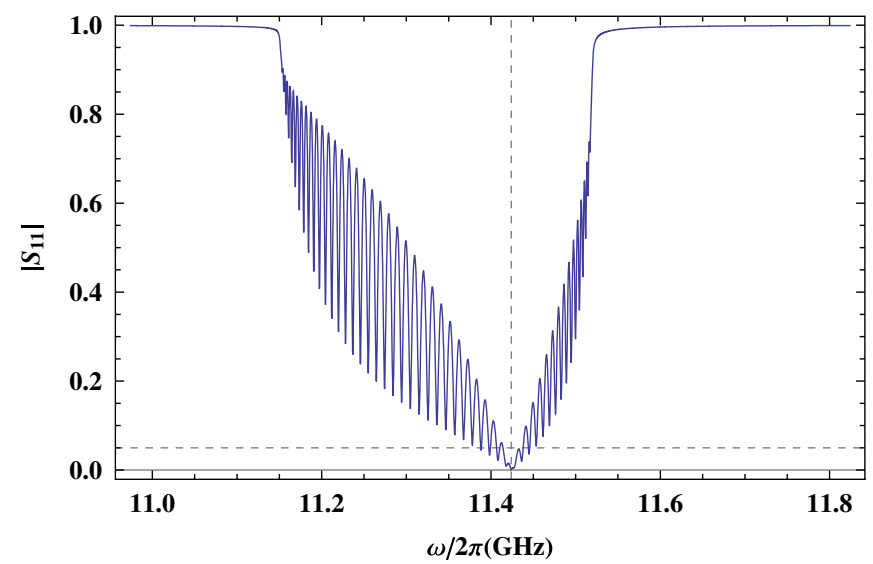

FIG. 5. (Color) Absolute value of reflection coefficient after tuning input and output cells for CZ120VG3 viewed from input port.

the direct method gives the reflection coefficient as viewed from the input port and this is illustrated in Fig. 5. The vertical dashed line indicates the location of the accelerat- ing frequency. The horizontal dashed line indicates the reflection at 0.05 and this corresponds to an approximately $1 \%$ bandwidth.

Using the circuit model, we then launch a pulse through this accelerating structure for a pulse with parameters similar to the CLIC pulse flattop length (the present design has 312 bunches spaced from each other by $0.5 \mathrm{~ns}$ ). This pulse has a flattop of $156 \mathrm{~ns}$ and a finite rise time of $10 \mathrm{~ns}$. The progress of the pulse through the accelerating structure is monitored by taking the convolution of the time response of the circuit with the pulse shape. The results of this simulation are displayed in Fig. 6. It is clear that even for the structure with a $2 \pi / 3$ phase advance per cell minimal pulse distortion occurs. We confirmed these simulations with the code SMART2D which includes higher order monopole bands but we obtained extremely similar results. Additional simulations made with shorter pulse widths down to $100 \mathrm{~ns}$ also indicate minimal distortion in the shape of the pulse.

We proceeded with other simulations of pulse propagation through a structure known as H60VG3 which consists
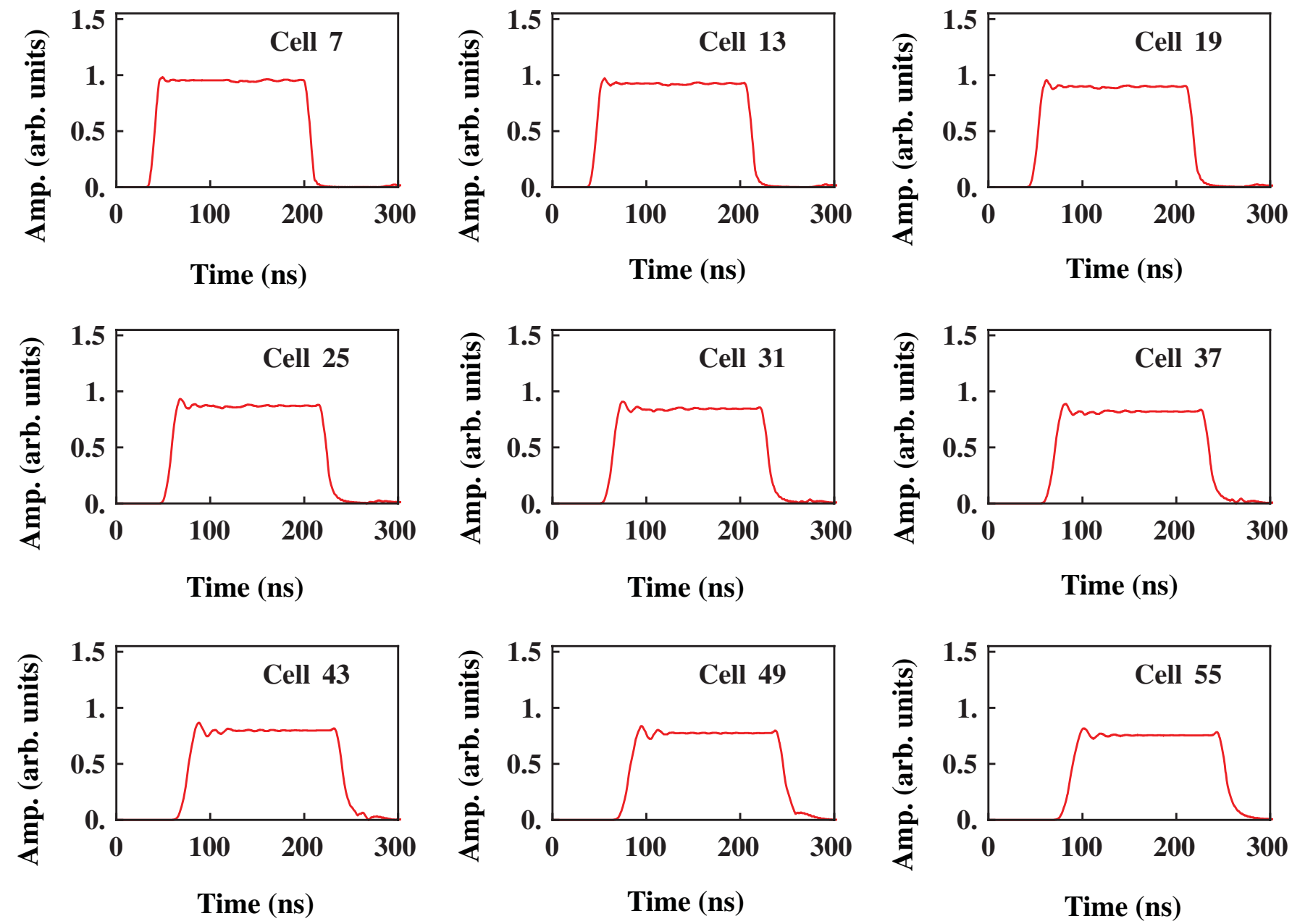

FIG. 6. (Color) Propagation of a rectangular pulse through a 55 cell structure CZ120VG3. The pulse is shown at various cells down the structure. 
of 55 cells with a nonconstant impedance. The phase advance per cell in this structure is $5 \pi / 6$ and the average group velocity of the fundamental mode is $0.03 c$. This structure is designed to suppress the beam-excited higher order modes (HOMs) by detuning the frequencies of these modes [18] and this leads to a structure in which the irises taper down along the accelerator. Although in practice detuning the cell frequencies is not sufficient and in addition, manifold damping is incorporated in the HOM wakefield suppression [18]. The potential for excitation of nearby monopole modes is minimized by ensuring the dimensional tolerances are strictly adhered to in machining and, subsequently, diffusion bonding of the cells. Indeed, the geometry of this structure was carefully designed and fabricated to achieve dimensional tolerances accurate to a few $\mu \mathrm{m}$. It is impractical to model the propagation of the rf pulse through the system by taking into account each individual feature in the geometry using finite element or finite difference electromagnetic codes [19-21] as it does not readily lend itself to modifying the cell geometries.

In order to achieve a rapid simulation of the essential dispersion characteristics of the structure, we modeled it by approximating the cells with abrupt transitions using SMART2D. We varied the cell iris and cavity radius in order to match the 0 and $\pi$ phase advance points for every cell subjected to infinite periodicity conditions. We then verified the accuracy of this technique with the wellestablished code KN7C [22]. The Brillouin diagram for a representative sample of cells from the structure is illustrated in Fig. 7(a) and the corresponding group velocity at the designed phase advance in Fig. 8(b). As the structure is designed to operate at a phase advance per cell of $150^{\circ}$ then the curves of all cells must pass through this point at the operating accelerating frequency and this is indicated by the dashed lines. We performed a similar matching procedure as was done with CZ120VG3 using the Kroll method and obtained a mean interior reflection coefficient
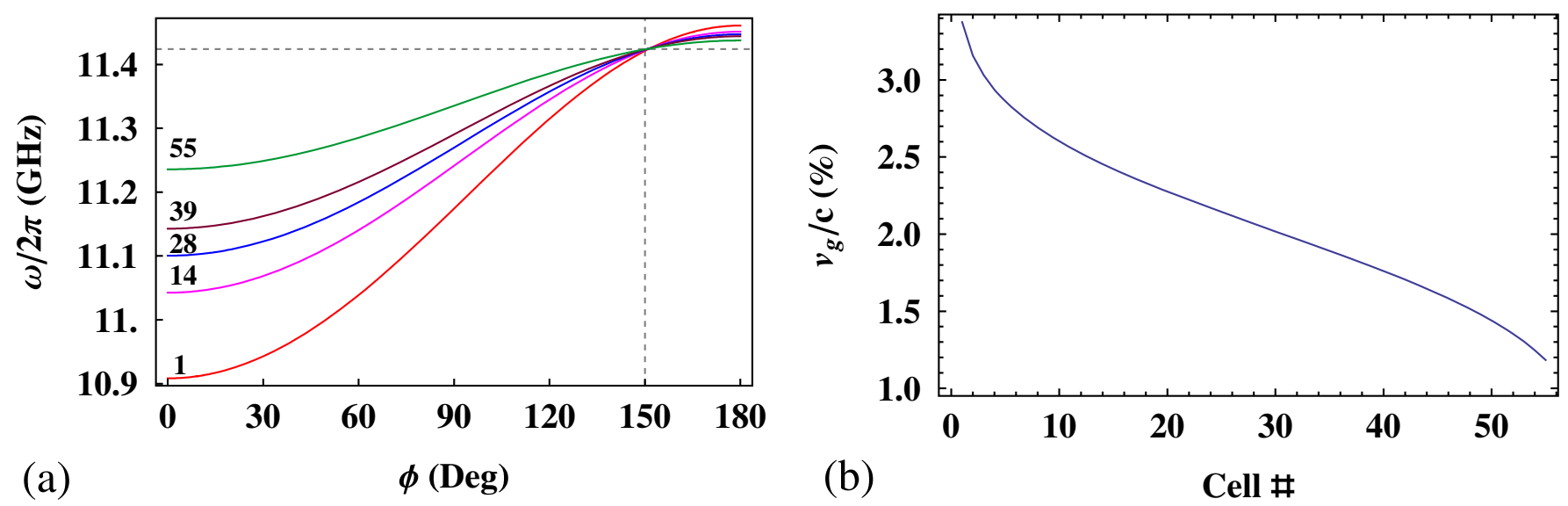

FIG. 7. (Color) Brillouin diagram (a) and group velocity (b) of H60VG3 cells designed with SMART2D.
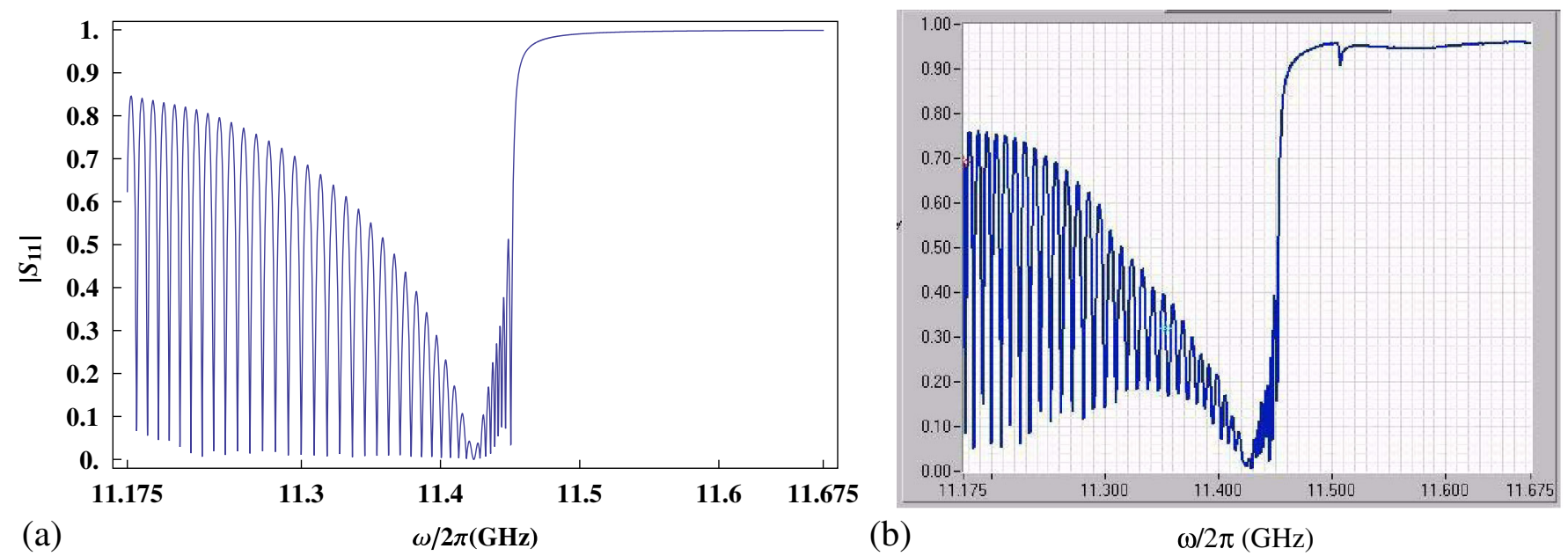

FIG. 8. (Color) Simulation (a) and experimental measurement (b) of reflection coefficient $S_{11}$ for H60VG3. 
of $-66 \mathrm{~dB}$. We also evaluated the resulting reflection from the input port. This reflection coefficient is displayed in Fig. 8(a) and this bears comparison to an experimental measurement on the structure made after tuning the structure, illustrated alongside in Fig. 8(b). Ohmic losses, corresponding to dissipation in the walls of the cavity, are not included in the simulation and this may account for the reason that almost all the signal is reflected for frequencies larger than $11.5 \mathrm{GHz}$, whereas in the experiment the reflection coefficient is below unity in this region. Indeed, the overall envelope of the signal observed in the experiment is a little smaller than that obtained in the simulation. The progress of the propagation of a rectangular pulse with a $400 \mathrm{~ns}$ flattop and a $10 \mathrm{~ns}$ rise and fall time through the cavity is illustrated Fig. 9. Here it is clear that the dispersion is large enough to give rise to appreciable distortion in the shape of the pulse. The resulting ripple in the field is expected to cause an energy spread in the energy gained by the train of accelerated bunches in a linear collider and this will be discussed in the next section. We also propagated a pulse through the structure in the Next Linear Collider Test Accelerator (NLCTA [23]) facility as part of tests on the high-gradient operation of these cavities. A typical experimental pulse shape, initially rectangular with a bandwidth of $240 \mathrm{~ns}$ and with a rise and fall time of $10 \mathrm{~ns}$, is illustrated Fig. 10 together with the results of a circuit model simulation in Fig. 10. The essential features of the ripple observed in the pulse are quite well represented with the circuit model employed. These results, indicating significant pulse distortion in the high phase advance structures, are confirmed by considering the dispersion parameter $D$ and are discussed in the Appendix.

In the next section we simulate a specific pulse shape propagating through an accelerating structure with the effect of beam loading also taken into account. The latter effect occurs due to the bunches in the train removing energy from the accelerating electric field and hence loading down the overall field. This total gradient will correspond to the energy gradient experienced by the accelerated train of bunches.

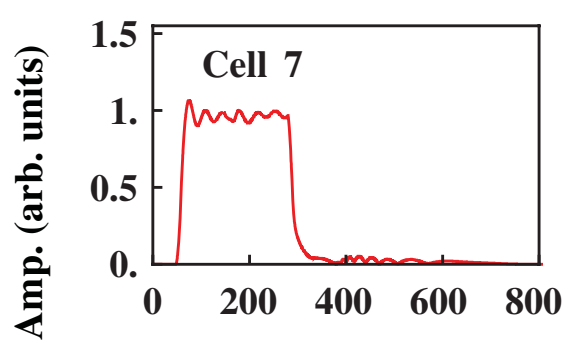

Time (ns)

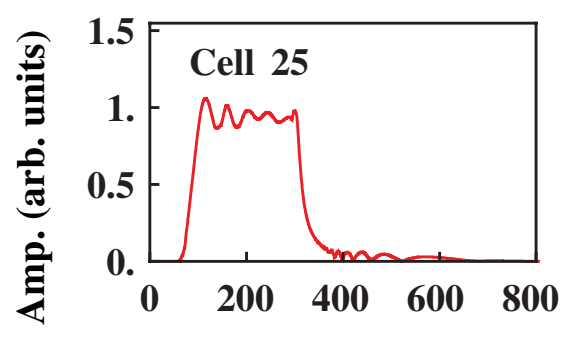

Time (ns)

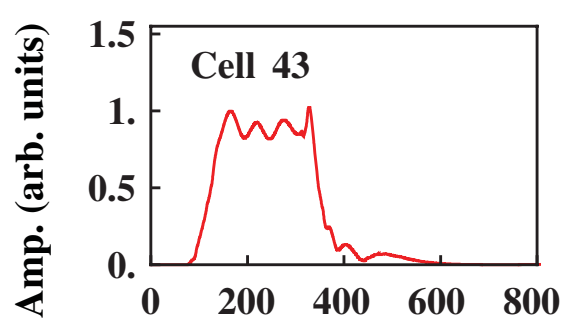

Time (ns)

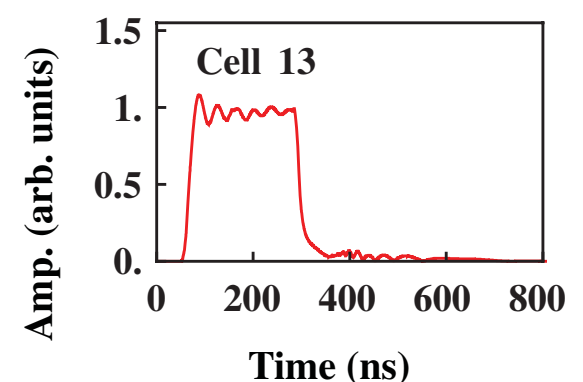

Time (ns)

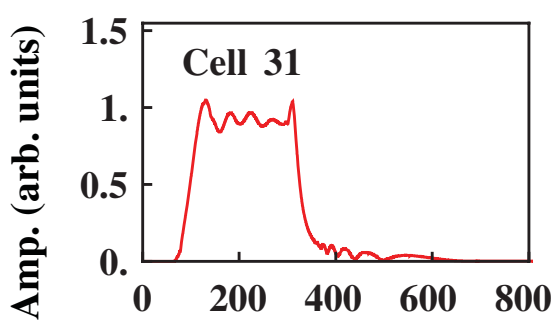

Time (ns)

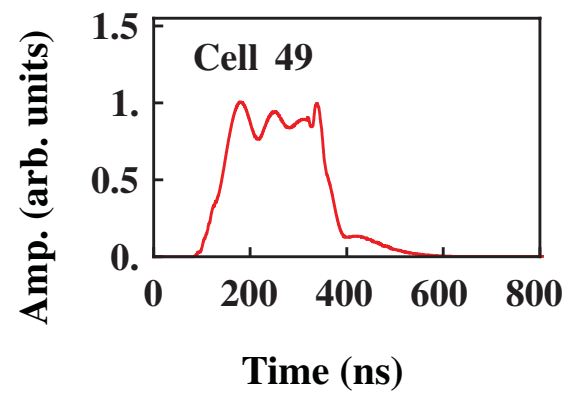

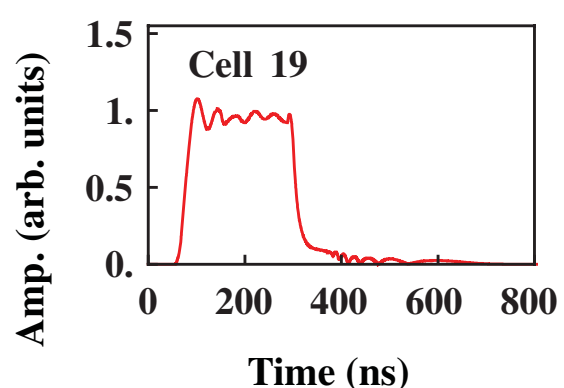

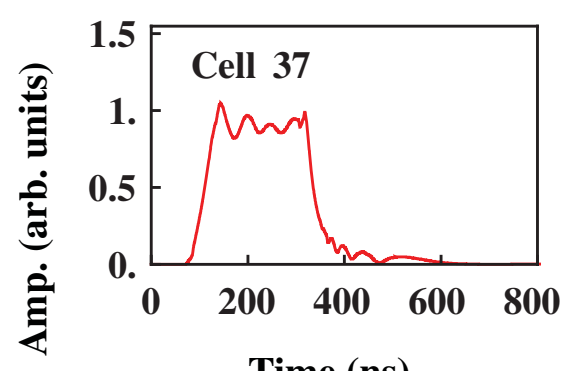

Time (ns)

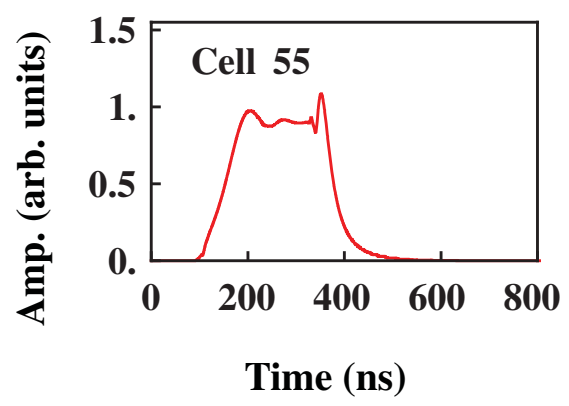

FIG. 9. (Color) Propagation of a rectangular pulse through a $60 \mathrm{~cm}$ structure H60VG3. The pulse is shown at various cells down the structure. 

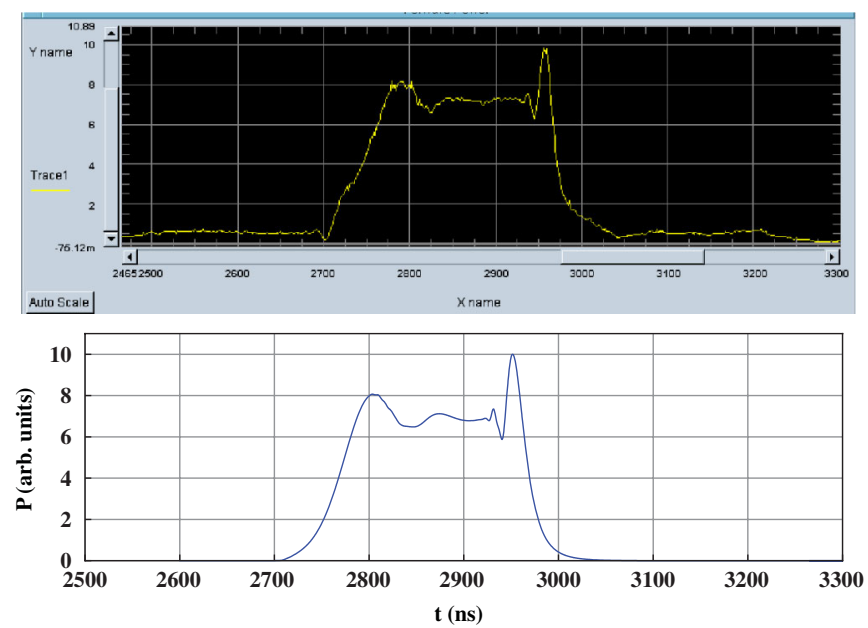

FIG. 10. (Color) Experimental (uppermost) and simulation (lowermost) of the power in a pulse after propagating through H60VG3.

\section{ENERGY DISPERSION AND BEAM LOADING}

In order to simulate the total energy gained by a train of charged particles, we used the code SMART2D to model both the characteristic impedance [13] of the accelerating structure and the transmission function of the structure. The latter was also simulated in the previous section with the circuit model and the result of these independent methods compare well. Here we again study the structure H60VG3 and we will analyze the effect of accelerating a train of 192 bunches spaced from their neighbors by $1.4 \mathrm{~ns}$. This structure and associated beam parameters correspond to that contemplated for use in the NLC [4]. We model H60VG3 with the code SMART2D. This code is able to calculate both the transmission and impedance properties of sharp transitions in the waveguide. For example, a single accelerating cell is modeled as a transition from wide to narrow to wide waveguide (WNW); the narrow region corresponds to the iris. The 0 and $\pi$ phase advance frequencies are matched to that of seven representative cells obtained from OMEGA2D simulations by varying the radius of the WN transitions. Intermediate cells are then obtained by spline interpolation. In this manner the complete accelerating structure is built up from WNW transitions and is illustrated in Fig. 11. The accelerating structure was then matched at each end to ensure optimal transmission in the vicinity of the accelerating frequency and to properly model the scattering matrix properties of the overall structure as fabricated. The matching was achieved by calculating $S_{11}$ the reflection coefficient of the overall structure at five frequency points in the vicinity of the accelerating frequency. $S_{11}$ was minimized by varying the iris and cavity radius of the end cells until an adequate match was obtained. A similar procedure was followed [24] to study the dipole characteristics of the structure in order to

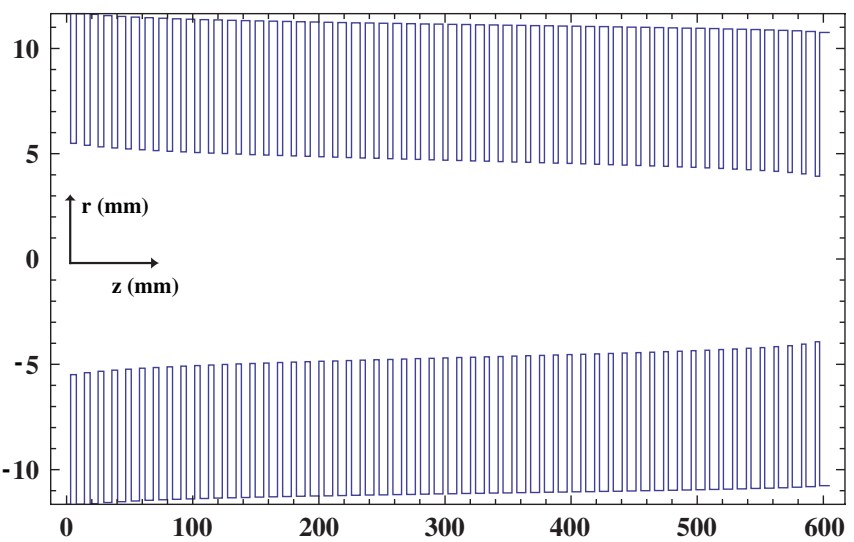

FIG. 11. (Color) Geometrical configuration of a series of WNW transitions used to model H60VG3.

understand the momentum kick the beam experiences due to higher band transverse wakefields.

The impedance $Z$ and the transmission function $G$ of the structure were then calculated with SMART2D and is illustrated in Fig. 12. Provided the structure has been designed correctly, both functions should be at a peak value at the accelerating frequency and this is indeed the case. The integral of the impedance $Z$ over all frequencies enables the loss factor to be obtained and this evaluates to $1.764 \mathrm{~V} / \mathrm{pC}$. This compares well with $1.762 \mathrm{~V} / \mathrm{pC}$ which is the average value of all those used in the previous section and was obtained from OMEGA2D [20] simulations. The inverse Fourier transform of the impedance enables the single-bunch wake function to be obtained, and summing this over the complete multibunch train gives the loaded gradient experienced by the accelerated beam.

We propagate a pulse through the H60VG3 structure which has an initial rise and fall time of $10 \mathrm{~ns}$ and is provided with a linear ramp (to compensate for the droop encountered in beam loading) of $100 \mathrm{~ns}$ in a gradient difference of $30 \mathrm{MV} / \mathrm{m}$. Various components of this pulse

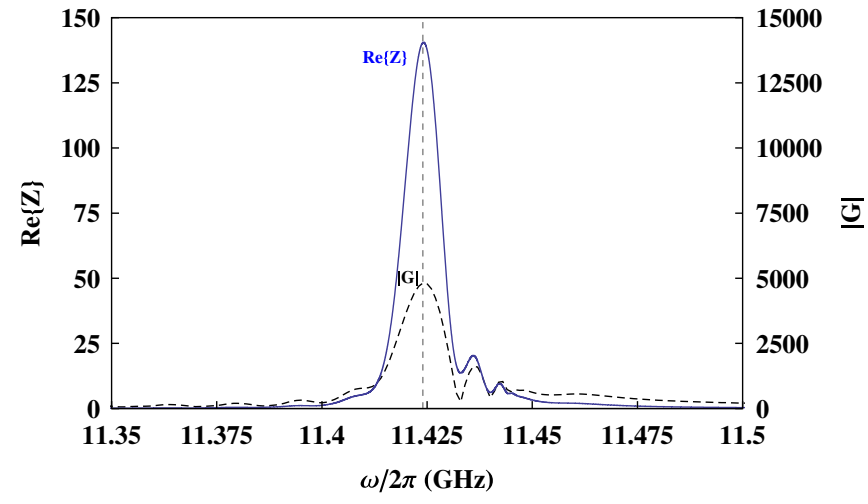

FIG. 12. (Color) Impedance ( $Z$, the solid blue line) and transmission function ( $G$, the dashed black line) of H60VG3. The dashed vertical line indicates the frequency of the accelerating mode. 


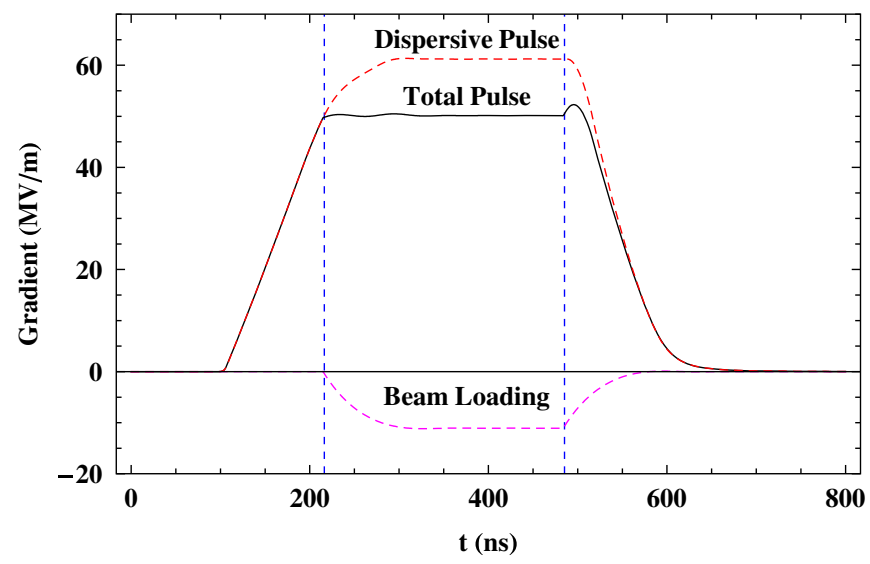

FIG. 13. (Color) Individual components of a beam-loaded pulse after propagation through H60VG3. The dashed vertical lines indicate the temporal location of the bunch train.

are indicated in Fig. 13. The total pulse incorporates both the propagation of the pulse through the structure including dispersive effects and the influence of beam loading. The average accelerating field gradient along the pulse is $\sim 50.4 \mathrm{MV} / \mathrm{m}$ and the average electric field contributed from beam loading is $\sim-10.8 \mathrm{MV} / \mathrm{m}$. Dispersive effects modify the degree of flatness of the total pulse shape. In order to investigate this, the region indicated by the vertical dashed lines, corresponding to the location of the bunch train, is amplified and displayed in Fig. 14 by the solid curve 0 . The energy deviation over the bunch train is displayed and it reaches a peak value of approximately $0.75 \%$, with an rms value deviation along the train of $0.22 \%$. In order to compensate for this energy deviation along the train, we add a component of the derivative of the energy deviation along the train to the original input $\mathrm{rf}$ pulse shape and minimize the resulting energy deviation along the train by making an optimal choice for the location of this added component to the input pulse. We wrote code which performs this optimization. The result of this

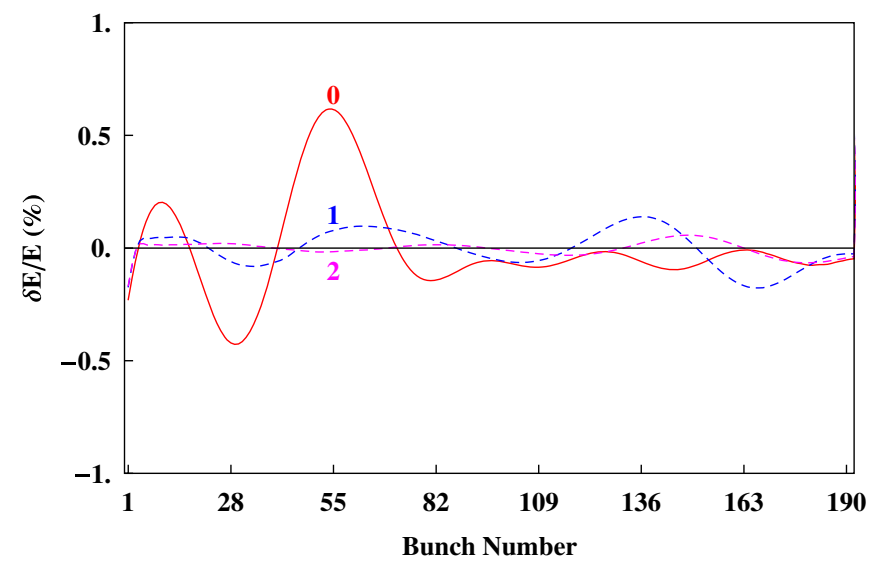

FIG. 14. (Color) Energy deviation over bunch train for no compensation (0) and for various stages of iterative compensation.

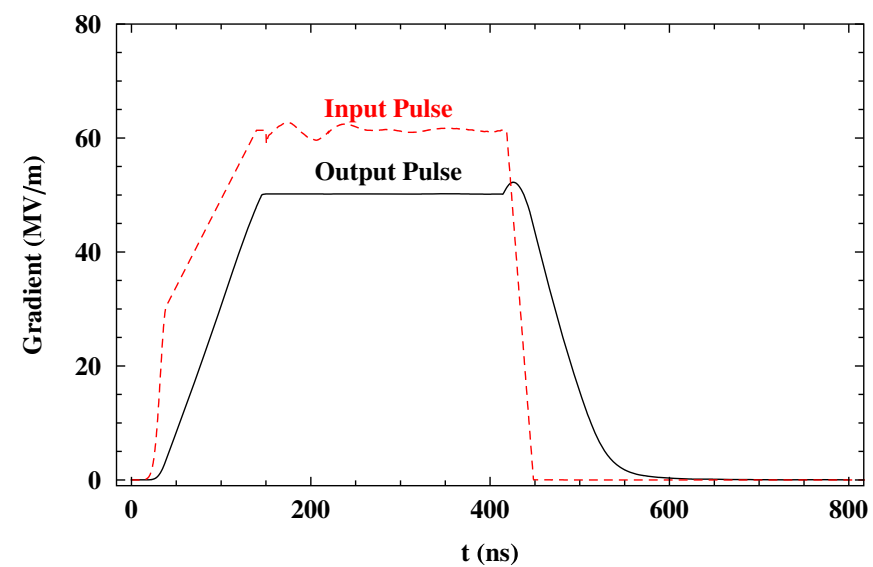

FIG. 15. (Color) Final input pulse shape (shown dashed in red) required to compensate energy deviation over bunch train. Also shown is the output pulse after compensation.

optimization is illustrated in Fig. 14 by the dashed curve 1 and for this case the maximal deviation in the energy is reduced to $0.13 \%$ with a $\mathrm{rms}$ deviation along the train of $0.08 \%$. We continue with this process to take the new optimized pulse shape and add further components of the energy deviation in order to minimize the overall energy deviation along the train. Indeed, only one additional iteration is necessary in order to reduce the maximum energy deviation along the train to no more than $0.05 \%$. The corresponding input pulse shape required for this energy compensation scheme is illustrated in Fig. 15 together with the resulting output pulse shape.

Finally, it is worth noting that the energy compensation scheme suggested here is not unique and other methods can be expected to give similar results on reducing the overall energy spread. For example, it may be possible to make a small perturbation to the input pulse shape over the ramp of the initial pulse shape to achieve similar compensation.

\section{CONCLUSIONS}

The circuit model developed herein provides a good description of pulse propagation in multicell accelerating structures suitable for linear collider applications. Pulse propagation through the rather standard SLAC-style accelerating structures which have a $2 \pi / 3$ phase advance per cell shows minimal dispersion for a monopole mode group velocity as low as $0.03 c$. However, the higher phase advance structures such as H60VG3, operating with a $5 \pi / 6$ phase advance per cell, reveal a significant distortion in the pulse shape due to dispersion.

Further evidence on expected pulse distortion is provided by the dispersion parameter $D$, a measure of the severity of dispersion. This parameter is defined as the derivative of the reciprocal of group velocity with respect to wavelength [25-27]. This parameter provides a measure of the anticipated broadening or compression in the shape of a pulse propagating through the structure. As shown in 
the Appendix, close to $\pi$ phase advance per cell $D$ is maximized and the distortion in the pulse shape is exacerbated. These conclusions on the expected pulse distortion, based on $D$, have been confirmed by our simulations on pulse propagation through high phase advance structures.

The total pulse, including beam loading, has been simulated and a finite energy spread occurs due to dispersion for H60VG3, the structure originally designed for the NLC. Means to compensate for this energy dispersion have been suggested, such as shaping the input pulse by suitably modifying the phase of the klystron rf sources. Finally we note that these structures, although originally destined for use in the NLC linacs, are receiving renewed interest as their ability to sustain high gradients makes them suitable, with some modification, for operation of multi-TeV linacs in the CLIC lepton collider design.

\section{ACKNOWLEDGMENTS}

The overall program of $X$-band accelerators for linear colliders in the NLC/GLC scenario has benefited from seamless collaborations with the following laboratories: SLAC, KEK, Fermi, and LBNL. In particular, we would like to single out the important contributions made by several individuals: $S L A C-\mathrm{C}$. Adolphsen, N. Baboi, K. Bane, G. Bowden, D. L. Burke, J. Cornuelle, S. Doebert, Z. Farkas, J. Frisch, E. Garwin, S. Harvey, H. Hoag, K. Jobe, R. Kirby, N. Kroll, F. Le Pimpec, Z. Li, G. A. Loew, J. Lewandowski, R. J. Loewen, D. McCormick, R. H. Miller, C. Nantista, J. Nelson, C. K. Ng, R. Palmer, E. Paterson, C. Pearson, N. Phinny, T. Raubenheimer, M. Ross, R.D. Ruth, T. Smith, S. Tantawi, P. Tenenbaum K. Thompson, and P. B. Wilson; $K E K-$ H. Baba, Y. Funahashi, N. Higashi, Y. Higashi, N. Hitomi, H. Kawamata, N. Kudo, T. Kume, H. Matsumoto, Y. Morozumi, J. S. Oh, T. Shintake, K. Takata, T. Takatomi, N. Toge, K. Ueno, and Y. Watanabe; FNAL-T. Arkan, C. Boffo, H. Carter, D. Finley, I. Gonin, T. Khabiboulline, S. Mishra, G. Romanov, N. Solyak; $L L N L-J$. Klingmann and K. van Bibber. This work was supported by the U.S. Department of Energy under Contract No. DE-AC0276 SF00515.

\section{APPENDIX: DISPERSION PARAMETER}

The time delay through an accelerating section is proportional to the reciprocal of the group velocity. In order to study the dependence on wavelength it is natural to define $D$, a dispersive parameter, in terms of the derivative of the reciprocal of the group velocity with respect to wavelength [25-27]:

$$
D=\frac{d}{d \lambda}\left(\frac{1}{v_{g}}\right)=\frac{1}{v_{g}^{2}} \frac{\omega^{2}}{2 \pi c} \frac{d v_{g}}{d \omega}=\frac{\omega^{2}}{2 \pi c P} \frac{d^{2} \omega}{d \phi^{2}}\left(\frac{d \phi}{d \omega}\right)^{3} .
$$

This parameter, in units of DP, is displayed in Fig. 16 for

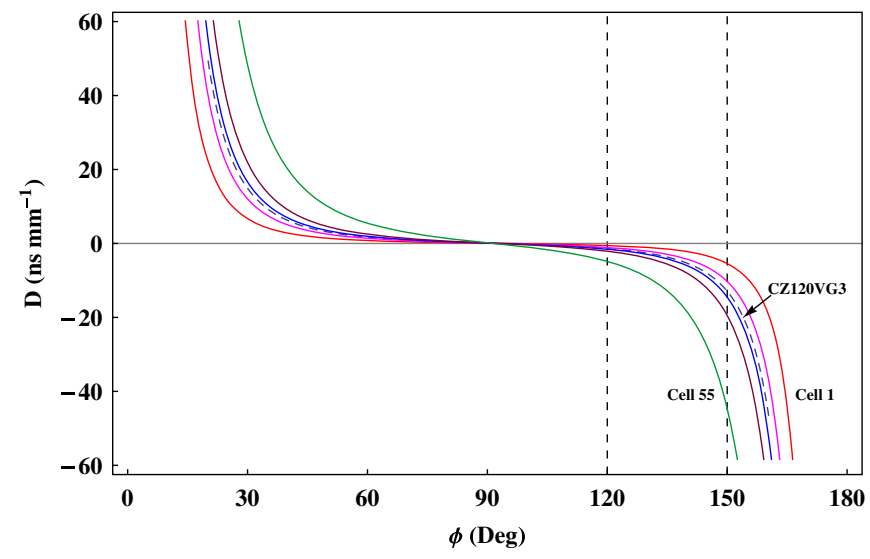

FIG. 16. (Color) Dispersive parameter DP for H60VG3 (cells 1, 14, 28, 39, and 55) and for CZ120VG3. The design phase advance of 150 and 120 degrees, for each of these structures, is indicated by the vertical dashed lines.

the structure H60VG3 (for the corresponding cells in Fig. 6) and for CZ120VG3 (indicated by the black dashed curve). At the phase advance 120 degrees, CZ120VG3 indicates a weak dispersive parameter of $\sim-1.4 \mathrm{~ns} / \mathrm{mm}$. However, in H60VG3 at the design phase advance of 150 degrees, the group velocity varies from $0.01 c$ to $0.03 c$ and in this case the dispersive parameter is relatively large, ranging from $\sim-5.4$ to $-44.6 \mathrm{~ns} / \mathrm{mm}$. This indicates that the dispersive effects are $\sim 30$ times larger in the high phase advance structure, H60VG3 compared to the conventional structure, CZ120VG3. These results confirm the strong dependence on group velocity seen in the simulations of pulse propagation through these structures.

[1] International Linear Collider Technical Review Committee (ILC-TRC) Second Report, SLAC-R-606, 2003.

[2] International Linear Collider Technical Review Final Report, 2004 (http://www.linearcollider.org).

[3] The TESLA Technical Design Report, edited by R. Brinkmann et al., DESY Report No. 2001-33, 2001.

[4] 2001 Report on the Next Linear Collider, edited by N. Phinney, SLAC-R-571, 2001.

[5] H. Braun et al., Report No. CLIC-Note-764, 2008.

[6] F. Tecker (CLIC and CTF3 Collaborations), J. Phys. Conf. Ser. 110, 112005 (2008).

[7] P. B. Wilson (private communication).

[8] The Stanford Two-Mile Accelerator, edited by R. B. Neal (W. A. Benjamin, Inc., New York, 1968).

[9] G. Geschonke et al., CLIC Note 45, 1987.

[10] G. Guignard and J. Hagel, Phys. Rev. ST Accel. Beams 3, 042001 (2000).

[11] G. Riddone (private communication).

[12] W. Wuensch, Proceedings of the X-band Structures and Beam Dynamics Workshop (XB08) (Cockcroft Institute, Daresbury, UK, 2008). 
[13] P. B. Wilson, contribution to the U.S. Particle Accelerator School, Batavia, IL, Report No. SLAC-PUB-4547, 1987.

[14] V.A. Dolgashev, presented at the International Computational Accelerator Physics Conference (ICAP), Monterey, CA, 1998.

[15] T. Itoh, Numerical Techniques for Microwave and Millimeter-Wave Passive Structures (John Wiley and Sons, New York, 1989).

[16] R. M. Jones et al., in Proceedings of the 8th European Particle Accelerator Conference, Paris, 2002 (EPS-IGA and CERN, Geneva, 2002).

[17] N. M. Kroll et al., Proceedings of the 20th International Linac Conference, Monterey, CA, 2000 (SLAC, Menlo Park, CA, 2000).

[18] R. M. Jones et al., Phys. Rev. ST Accel. Beams 9, 102001 (2006).

[19] MAFIA Collaboration, MAFIA-L, CST, GmbH, Darmstadt, 1994.

[20] Z. Li et al., in Proceedings of the 9th European Particle Accelerator Conference, Lucerne, 2004 (EPS-AG,
Lucerne, 2004).

[21] W. Bruns, in Proceedings of the Particle Accelerator Conference, Vancouver, BC, Canada, 1997 (IEEE, New York, 1997).

[22] E. Keil, Nucl. Instrum. Methods 100, 419 (1972).

[23] R. D. Ruth et al., in Proceedings of European Committee for Future Accelerators Workshop on $e^{+} e^{-}$Linear Colliders, Garmisch Partenkirchen, Germany, 1992; also SLAC Report No. SLAC-PUB-6293.

[24] R. M. Jones, in Proceedings of the 22nd Linear Accelerator Conference (LINAC 2004), Lübeck, Germany, 2004.

[25] R. Ramaswami and K. N. Sivarajan, Optical Networks: A Practical Perspective (Academic Press, London, 1998).

[26] A. Martınez, A. Garcia, P. Sanchis, and J. Martı, J. Opt. Soc. Am. A 20, 147 (2003).

[27] K. Saitoh et al., New J. Phys. 8, 207 (2006).

[28] J. W. Wang, Ref. [12]. 